\title{
Prolonged anticoagulation in VTE with direct oral anticoagulants: towards an individual analysis of net clinical benefit?
}

Pierre-Marie Roy

Affiliation: Emergency Dept, CHU Angers, and Institut MITOVASC, EA 3860, Université d'Angers, Angers, France.

Correspondence: Pierre-Marie Roy, CHU d'Angers, Département de Médecine d'Urgence, Centre Vasculaire et de la Coagulation, 4 rue Larrey, 49933 Angers Cedex 09, France. E-mail: PMRoylachu-angers.fr

0 @ERSpublications

Beside scores, doctor-patient relationship still key to assess individual benefit of extended anticoagulation http://ow.ly/uWaL303tb77

Venous thromboembolism (VTE) is a common, multifactorial and capricious disease. Its incidence is estimated at between 1 and 1.84 per 1000 person-years, half of cases consisting of isolated distal or proximal deep vein thrombosis (DVT), a quarter of isolated pulmonary embolism (PE) and the rest being $\mathrm{PE}$ associated with DVT $[1,2]$. The direct severity depends on the mortality risk due to haemodynamic instability in massive PE, and risk of mid- or long-term thromboembolism recurrence and associated sequelae [3]. Population-based studies have revealed that the recurrence risk is significant in the first days, intermediate during the next 3-6 months, then falls to approximately 3-5\% per year, though varies widely depending on the thromboembolism context and patient characteristics [1, 3]. We can thus distinguish three therapeutic stages: initial treatment (acute phase), maintenance treatment (consolidation phase) and, at least for some patients, long-term treatment (secondary prevention phase) [4-6].

The emergence of direct oral anticoagulants (DOACs) has made a profound difference in each stage of the process. Initially prescribed orally (rivaroxaban and apixaban) or following a few days of administering parenteral anticoagulant (dabigatran and edoxaban), these agents enable avoiding overlapped treatment phases, which carries a particular bleeding risk with vitamin $\mathrm{K}$ antagonists (VKAs) [7]. They also offer greater, wider treatment windows than VKAs, thus generally proving noninferior to LMWH/VKA combinations in terms of efficacy, yet superior in terms of safety. This positive effect was confirmed in a meta-analysis reviewing five studies and involving nearly 25000 patients, reporting a $40 \%$ reduction in relative risk (RR) of major bleeding ( $R R$ $0.60,95 \%$ CI $0.41-0.88$ ) and $>60 \%$ reduction in risk of fatal bleeding (RR 0.36, 95\% CI 0.15-0.87) [8]. Nevertheless, managing bleeding used to be a serious concern until consensus approaches were established $[9,10]$ and antidotes were discovered $[11,12]$. The latter are now recommended as first-line in the latest international guidelines for acute VTE [5]. They are also indicated in secondary prevention, though this is more complex due to the very fine line between thrombosis and bleeding risks in this context [13]. Two approaches are therefore necessary to define which patients are likely to benefit from long-term DOACs as secondary prevention: identifying first those at high risk of recurrence, then those at low bleeding risk.

The study by KLoK et al. [14], published in this issue of the European Respiratory Journal, addresses this using data from two randomised studies comparing dabigatran with warfarin in the management of proximal acute VTE. In those patients randomly assigned to the dabigatran arm, six factors were shown to be independent predictors of severe bleeding: active cancer, being male with uncontrolled hypertension, anaemia, history of bleeding, renal dysfunction, and age. The authors integrated them into the VTE-BLEED risk score, then analysed its prediction performance for severe bleeding risk after 1 month of treatment (once achieving therapeutic balance). Patients with VTE-BLEED scores $\geqslant 2$ exhibited a 6.5 times

Received: Aug 182016 | Accepted: Aug 192016

Conflict of interest: Disclosures can be found alongside this article at erj.ersjournals.com

Copyright OERS 2016 
higher severe bleeding risk with either dabigatran (incidence $1.4 \%$ versus $0.22 \%$ ) or VKAs (2.8\% versus $0.44 \%)$. VTE-BLEED scores thus enable stratification of patient haemorrhage risk with dabigatran or VKAs after the acute phase, which, as the authors suggest, can be helpful in the decision-making process when considering prolonging anticoagulation.

This work displayed limitations, notably owing to its population, and requires assessment and validation by prospective studies on less-targeted populations, especially involving those contraindicated for dabigatran, for example, severe kidney failure patients, and those receiving other DOACs. Furthermore, if according to VTE-BLEED, the relative risk of bleeding is similar with VKAs and dabigatran, the absolute risk differs greatly. Such differences may be observed with and between other DOACs $[15,16]$. Nevertheless, the important finding of this study is that a simple score, assessed during the consolidation phase, may be suitable for bleeding-risk assessment for the secondary prevention phase, at least for dabigatran [17].

All in all, the main question I, as a physician, would ask is "how can I use VTE-BLEED or another haemorrhage risk score to opt for or against proposing prolonged anticoagulants to a patient?". To answer this, the treatment-related bleeding risk must be assessed in terms of potential benefit, i.e., the thrombosis recurrence risk [18]. When VTE occurs in the absence of major transient triggering factors like surgery, severe trauma or immobilisation due to acute illness, this risk is generally high at a later time point (10-25\% at 1 year and $30-40 \%$ at 5 years depending on studies) [19-21]. Excluding cancer patients, this represents $40-60 \%$ of patients $[1,2]$ but only one third may benefit from extended treatment. The challenge is thus to stratify this heterogeneous population so as to identify those at high or low risk of recurrence. Several scores or clinical rules have been proposed for this end $[22,23]$. Among them, the "men continue and HERDOO 2" rule was recently validated in an interventional study. Women who discontinued anticoagulation and had one or fewer HERDOO criteria (hyperpigmentation, oedema, or redness on examination in either leg; D-dimer $>250 \mathrm{ng} \cdot \mathrm{mL}^{-1}$ (Vidas; BioMérieux, Marcy l'Etoile, France); obesity (body mass index $>30 \mathrm{~kg} \cdot \mathrm{m}^{-2}$ ); and older age ( $>65$ years)) had lower risk of recurrent VTE (approximately $3 \%$ per patient-year of follow-up) as did high-risk patients who continued anticoagulation [24].

As for whether this means that one could simply weigh VTE-BLEED against HERDOO 2 scores to assess the benefit/risk ratio of prolonged anticoagulation following unprovoked VTE, the answer is undoubtedly no. Each score significantly simplifies the reality by only considering a very limited number of categorical or dichotomised parameters like age or kidney function, also notably grouping together all haemorrhagic complications as one and all thromboembolism recurrences as another. Thus gastrointestinal bleeding requiring transfusion and intracranial haemorrhage are both categorised as severe bleedings, despite their treatment possibilities and consequences for the patient being vastly different and, most importantly, each possessing different risk factors [25]. Arterial hypertension increases the risk of intracranial haemorrhage, contrary to previous gastrointestinal bleeding with ulcers [26]. Similarly, the risk of recurrence under the clinical presentation of $\mathrm{PE}$ is much higher for those patients having manifested prior PE during a first thromboembolism episode than for those initially presenting with isolated DVT. Yet, for a similar risk of recurrent VTE, the case-fatality rate is four times greater after PE than after DVT [27]. Furthermore, assessing the benefit-risk ratio of prolonged anticoagulation using the VTE-BLEED and HERDOO 2 scores would again require attributing the same weight to any thromboembolic recurrence as to severe bleeding. This approach, aimed at assessing the net benefit of a treatment, would only be accurate when using a unique and unquestionable criterion, like lifespan or autonomous lifespan. Under such conditions, it would be highly likely that the elements and their weights differ greatly from those of VTE-BLEED and HERDOO 2 combined. Ideally, this global score should also evolve, integrating potential interactions over time between previous treatment length, treatment modalities, patient age and other parameters.

It is more than likely that using extremely large databases will enable us to, one day, design a method it is not a global score but rather a complex algorithm integrating all these data in a dynamic system, enabling precise modelling of risk/benefit ratios of prolonged anticoagulation, like the models for calculating the probabilities of internet shopping. There is one major element that appears, to me, impossible to model, however: the patient's perception, the anxiety they may have felt during the first episode, and their fear of recurrence or, conversely, of cerebral haemorrhage and the impact of avoiding haemorrhage-risk situations. While these scores may prove beneficial, it is the individual doctor-patient relationship and follow-up, as well as the physicians' role of listener as much as educator of the disease determinants, which would appear to be the key elements to understanding the net individual benefit and defining the optimal treatment adjusted to each patient.

\section{References}

1 Heit JA, Spencer FA, White RH. The epidemiology of venous thromboembolism. J Thromb Thrombolysis 2016; 41: 3-14.

2 Delluc A, Tromeur C, Le Ven F, et al. Current evidence of venous thromboembolism and comparison with 1998: a community-based study in Western France. Thromb Haemost 2016 [in press DOI: 10.1160/TH16-03-0205].

3 Kearon C. Natural history of venous thromboembolism. Circulation 2003; 107: 23: Suppl. 1, I22-I30. 
Goldhaber SZ, Bounameaux H. Pulmonary embolism and deep vein thrombosis. The Lancet 2012; 379: $1835-1846$.

5 Kearon C, Akl EA, Ornelas J, et al. Antithrombotic therapy for VTE disease: CHEST Guideline and Expert Panel Report. Chest 2016; 149: 315-352.

6 Konstantinides SV, Torbicki A, Agnelli G, et al. 2014 ESC guidelines on the diagnosis and management of acute pulmonary embolism. Eur Heart J 2014; 35: 3033-3069.

7 Heit JA, Lahr BD, Petterson TM, et al. Heparin and warfarin anticoagulation intensity as predictors of recurrence after deep vein thrombosis or pulmonary embolism: a population-based cohort study. Blood 2011; 118: 4992-4999.

8 van der Hulle T, Kooiman J, den Exter PL, et al. Effectiveness and safety of novel oral anticoagulants as compared with vitamin $\mathrm{K}$ antagonists in the treatment of acute symptomatic venous thromboembolism: a systematic review and meta-analysis. J Thromb Haemost 2014; 12: 320-328.

9 Pernod G, Albaladejo P, Godier A, et al. Management of major bleeding complications and emergency surgery in patients on long-term treatment with direct oral anticoagulants, thrombin or factor-Xa inhibitors: Proposals of the Working Group on Perioperative Haemostasis (GIHP) - March 2013. Arch Cardiovasc Dis 2013; 106: $382-393$.

10 Heidbuchel H, et al. Updated European Heart Rhythm Association Practical Guide on the use of non-vitamin K antagonist anticoagulants in patients with non-valvular atrial fibrillation. Europace 2015; 17: 1467-1507.

11 Pollack CV Jr, Reilly PA, Eikelboom J, et al. Idarucizumab for dabigatran reversal. N Engl J Med 2015; 373: 511-520.

12 Siegal DM, Curnutte JT, Connolly SJ, et al. Andexanet alfa for the reversal of factor Xa inhibitor activity. $N$ Engl J Med 2015; 373: 2413-2424.

13 Schulman S, Kearon C, Kakkar AK, et al. Extended use of dabigatran, warfarin, or placebo in venous thromboembolism. N Engl J Med 2013; 368: 709-718.

14 Klok FA, Hösel V, Clemens A, et al. Prediction of bleeding events in patients with venous thromboembolism on stable anticoagulation treatment. Eur Respir J 2016; 48: 1369-1376.

15 Castellucci LA, Cameron C, Le Gal G, et al. Efficacy and safety outcomes of oral anticoagulants and antiplatelet drugs in the secondary prevention of venous thromboembolism: systematic review and network meta-analysis. BMJ 2013; 347: f5133.

16 Di Nisio M, Ageno W, Rutjes AW, et al. Risk of major bleeding in patients with venous thromboembolism treated with rivaroxaban or with heparin and vitamin K antagonists. Thromb Haemost 2016; 115: 424-432.

17 Klok FA, Niemann C, Dellas C, et al. Performance of five different bleeding-prediction scores in patients with acute pulmonary embolism. J Thromb Thrombolysis 2016; 41: 312-320.

18 Couturaud F. Guided duration of anticoagulation after unprovoked venous thromboembolism using D-dimer testing. Eur Respir J 2016; 47: 1313-1314.

19 Couturaud F, Sanchez O, Pernod G, et al. Six months vs extended oral anticoagulation after a first episode of pulmonary embolism: the PADIS-PE randomized clinical trial. JAMA 2015; 314: 31-40.

20 Kearon C, Gent M, Hirsh J, et al. A comparison of three months of anticoagulation with extended anticoagulation for a first episode of idiopathic venous thromboembolism. N Engl J Med 1999; 340: 901-907.

21 Agnelli G, Prandoni P, Santamaria MG, et al. Three months versus one year of oral anticoagulant therapy for idiopathic deep venous thrombosis. N Engl J Med 2001; 345: 165-169.

22 Ensor J, Riley RD, Moore D, et al. Systematic review of prognostic models for recurrent venous thromboembolism (VTE) post-treatment of first unprovoked VTE. BMJ Open 2016; 6: e011190.

23 Palareti G, Cosmi B, Antonucci E, et al. Duration of anticoagulation after isolated pulmonary embolism. Eur Respir J 2016; 47: 1429-1435.

24 Rodger M. REVERSE II - Multi-national validation of the men continue and HERDOO2 Rule to identify low risk unprovoked thromboembolism patients who can discontinue anticoagulants. http://congress365.escardio.org/ Presentation/142492\#.V-AWgfkrKUk

25 Schulman S, Kearon C. Definition of major bleeding in clinical investigations of antihemostatic medicinal products in non-surgical patients. J Thromb Haemost 2005; 3: 692-694.

26 Ariesen MJ, Claus SP, Rinkel GJ, et al. Risk factors for intracerebral hemorrhage in the general population: a systematic review. Stroke 2003; 34: 2060-2065.

27 Carrier M, Le Gal G, Wells PS, et al. Systematic review: case-fatality rates of recurrent venous thromboembolism and major bleeding events among patients treated for venous thromboembolism. Ann Intern Med 2010; 152: $578-589$. 